\title{
Las posibilidades didácticas del WhatsApp en la formación docente no universitaria
}

The didactic possibilities of whatsapp in non-university teacher training

As possibilidades didáticas do WhatsApp na formação de professores não universitários

\section{Maciel Ortega ${ }^{1}$}

Universidad Nacional de Catamarca (Argentina)

Instituto Superior Clara J. Armstrong

https://orcid.org/0000-0001-8675-6885

D0I: https://doi.org/10.35622/j.rep.2021.01.002

Recibido 16/12/2020/ Aceptado 20/01/2021

RESUMEN. El trabajo sistematiza la experiencia de enseñanza desarrollada en una carrera de formación docente en tiempos de aislamiento social, la cual fue mediada por una aplicación para dispositivos móviles como es el WhatsApp. En el artículo se describe y sistematiza los usos didácticos del WhatsApp y las actividades de aprendizajes propuestas en la asignatura Sociología de la Educación del Profesorado en Educación Secundaria en Matemática de un Instituto de Educación Superior de la provincia de Catamarca. En el trabajo se adoptó una metodología cualitativa y se empleó para la recolección de datos, las clases elaboradas, la lectura y el análisis documental y a una encuesta semi estructurada aplicada a estudiantes. El trabajo pedagógico desarrollado con el WhatsApp, evidenció que la aplicación puede ser usada con fines didácticos, que sus utilidades pueden ser adaptadas para la enseñanza y que la incorporación de medios tecnológicos en la educación por sí mismos no generan innovación ni aprendizajes, sino que las estrategias de enseñanza y las tareas diseñadas, son las que favorecen o no la construcción de saberes y la autonomía en los aprendizajes².

PALABRAS CLAVE: educación superior, enseñanza, WhatsApp

ABSTRACT. The paper systematizes the teaching experience developed in a teacher training course in times of social isolation, which was mediated by an application for mobile devices such as WhatsApp. The article describes and systematizes the didactic uses of WhatsApp and the learning activities proposed in the Sociology of Education course of the Secondary Education Teacher's Degree in Mathematics at an Institute of Higher Education in the province of Catamarca. The work adopted a qualitative methodology and used for data collection, the elaborated classes, reading and documentary analysis and a semi-structured survey applied to students. The pedagogical work developed with WhatsApp showed that the application can be used for didactic purposes, that its utilities can be adapted for teaching and that the incorporation of technological means in education by themselves do not generate innovation or learning, but that the teaching strategies and the tasks designed are the ones that favor or not the construction of knowledge and autonomy in learning.

KEYWORDS: higher education, teaching, WhatsApp

\footnotetext{
${ }^{1}$ Correspondencia: telurio_vargas@yahoo.com.ar

${ }^{2}$ La experiencia se desarrolla en el marco del proyecto "Inclusión tecnológica y construcciones metodológicas en las prácticas de enseñanza del nivel superior. Aportes para reconstruir la Didáctica Universitaria”. Aprobado por la Secretaria de Investigación y Posgrado de la Universidad Nacional de Catamarca.
} 
RESUMO. 0 trabalho sistematiza a experiência docente desenvolvida em uma carreira de formação de professores em tempos de isolamento social, que foi mediada por um aplicativo para dispositivos móveis como o WhatsApp. 0 artigo descreve e sistematiza os usos didáticos do WhatsApp e as atividades de aprendizagem propostas na disciplina de Sociologia da Formação de Professores do Ensino Secundário em Matemática de um Instituto de Ensino Superior da província de Catamarca. No trabalho, foi adotada uma metodologia qualitativa e utilizada para coleta de dados, aulas, leitura e análise documental e uma pesquisa semiestruturada aplicada aos alunos. 0 trabalho pedagógico desenvolvido com 0 WhatsApp, mostrou que 0 aplicativo pode ser utilizado para fins didáticos, que suas utilidades podem ser adaptadas para o ensino e que a incorporação dos meios tecnológicos na educação por si só não gera inovação ou aprendizagem, mas sim as estratégias de Ensino as tarefas concebidas são aquelas que favorecem ou não a construção do conhecimento e autonomia na aprendizagem.

PALABRAS CLAVE: Ensino superior, ensino, WhatsApp.

\section{INTRODUCCIÓN}

La pandemia provocada por el Covid19, que actualmente azota al país, exigió que mediante Resolución No 108 del Ministerio de Educación de la Nación se dispusiera "la suspensión del dictado de clases presenciales en los niveles inicial, primario, secundario en todas sus modalidades, e institutos de educación superior" (2020), medida que recayó sobre todos los establecimientos educativos del país. Tal disposición demandó que los contenidos pedagógicos dejaran de impartirse desde las aulas escolares y se realizara su enseñanza mediante medios tecnológicos y plataformas virtuales que permitieran la interacción docente-alumnos y el acompañamiento en los aprendizajes. La pandemia planteó la necesidad de que los docentes implementaran prácticas educativas que tomaran en consideración, los contenidos curriculares a enseñar, las posibilidades de los estudiantes para apropiarse de los contenidos y el uso de medios en los procesos de enseñanza y aprendizaje, obligando con la inclusión de las tecnologías a "altera[r] propuestas, contenidos, formas de interactuar y evaluaciones, entre otros aspectos" (Maggio, 2018, p. 14), como también a implementar formas de enseñanza que antes no habían sido ensayadas. Desde la mirada de Cobos (2016), la aplicación de la tecnología en la educación propone el desafío de experiencias de enseñanza que favorezcan el aprendizaje, como también, el diseño de propuestas que no solo aspiren a la sistematización de los contenidos seleccionados, sino que inciten a la investigación como a la creatividad.

Las Tic en la enseñanza, posibilitan diferentes usos, entre los que se pueden mencionar "como instrumento de mediación entre los alumnos y el contenido o la tarea de aprendizaje (...), de representación y comunicación de significados sobre los contenidos o tareas (...), de seguimiento, regulación y control de la actividad conjunta de profesor y alumnos (...), [y] de configuración de entornos de aprendizaje y espacios de trabajo para profesores y alumnos" (Coll, Mauri Majós y Onrubia Goñi, 2008, pp. 9-11).

Las tecnologías favorecen el acceso a contenidos a través de los más variados formatos, permitiendo implementar prácticas y experiencias innovadoras que habiliten nuevas formas de aprender, formas que "promueven otros procesos cognitivos o capacidades 
comunicacionales" (España y Foresi, 2020, p. 189). En el nivel superior, el alto número de docentes y alumnos sin disposición de medios tecnológicos ni acceso a internet desde sus hogares, demandó la utilización de dispositivos móviles como el teléfono celular y aplicaciones como el WhatsApp y el correo electrónico, para desarrollar los contenidos y las actividades de enseñanza y aprendizaje. El WhatsApp es una aplicación móvil que haciendo uso de datos móviles, permite la formación de grupos y la comunicación sincrónica como asincrónica a través de mensajes de audio, texto, videos, enlaces a webs, fotos, llamadas, etc.

En la experiencia que se sistematiza, la aplicación fue utilizada en la enseñanza de Sociología de la Educación y manejada en un sentido pedagógico y didáctico que propició construcciones metodológicas (Edelstein, 1998) acordes a las posibilidades que ofrecía, buscando a partir de las propuestas diseñadas, alentar a los alumnos a aprender de forma autónoma (Maggio, 2016), mediante el involucramiento activo en actividades que propiciaran la construcción de nuevos aprendizajes. En el presente trabajo se busca exponer sobre: ¿Cuáles fueron los usos didácticos generados a partir de la inclusión del WhatsApp como herramienta de mediación tecnológica en la enseñanza de Sociología de la Educación? ¿Qué tipos de estrategias de enseñanza y actividades de aprendizajes fueron propuestos en la asignatura y de qué modo contribuyeron a la construcción de un aprendizaje autónomo? El objetivo del trabajo es describir y sistematizar, algunos de los usos pedagógicos- didácticos que se realizaron del WhatsApp y cómo su inclusión requirió el diseño de construcciones metodológicas, de actividades y formas de comunicación diferentes a las que impone la presencialidad. También, se busca poner en evidencia, que el WhatsApp puede ser usado con fines educativos y que sus utilidades pueden ser adaptadas para la enseñanza y que la incorporación de medios tecnológicos en la educación por sí mismos no generan innovación ni aprendizajes, sino que las estrategias de enseñanza propuestas y las tareas diseñadas, son las que favorecen o no la construcción de saberes.

\section{MÉTODO}

El presente trabajo consiste en una sistematización de experiencia centrada en un proceso de enseñanza mediado por tecnologías. Para la concreción del trabajo se adoptó una metodología cualitativa y se empleó como instrumento de recolección de datos a la lectura y el análisis de bibliografía referente a la enseñanza mediada por tecnologías, también se consideraron investigaciones publicadas en revistas científicas referidas al uso del WhatsApp en la enseñanza, las cuales fueron empleadas para establecer similitudes y diferencias entre los resultados obtenidos en éstas y los hallados en la experiencia que se sistematiza. Otros de los instrumentos de recolección que se emplearon fue el conjunto de documentos que se elaboraron para las clases, las que incluían síntesis explicativas de carácter conceptual y actividades de aprendizaje para los alumnos, y una encuesta semiestructurada aplicada a los estudiantes, a partir de la cual valoraron el uso del WhatsApp en la enseñanza, esta última fue aplicada vía correo electrónico, otra de las aplicaciones empleadas en la experiencia. 
En el trabajo se consideró como categoría de análisis a: 1) el uso de los medios tecnológicos en la enseñanza de la asignatura Sociología de la Educación, 2) las actividades de enseñanza y de aprendizajes dispensadas desde la asignatura y 3) las valoraciones realizadas por los estudiantes respecto del uso del WhatsApp en la enseñanza impartida.

Para el análisis de los datos se utilizó la técnica de análisis de contenidos desde una perspectiva cualitativa, la cual demandó la realización de lecturas de los documentos, de la encuesta y las clases, para la posterior agrupación de los datos según las categorías de análisis establecidas. Esta técnica de análisis exige la interpretación del contenido de los documentos, la identificación de fragmentos de los textos que estén vinculados a las categorías mencionada, para finalmente producir un nuevo escrito que articule los datos elaborados y analizados. La unidad de análisis que se definió para el procedimiento seguido con los documentos, fueron las líneas de textos que ofrecían referencias sobre las categorías delimitadas.

\section{MARCO NORMATIVO- ACADÉMICO DE LA EXPERIENCIA EDUCATIVA}

En Argentina, la formación de profesionales docentes, que se realiza en el marco de los institutos de educación superior, según la ley de Educación Nacional № 26.206, "tiene la finalidad de preparar profesionales capaces de enseñar, generar y transmitir los conocimientos y valores necesarios para la formación integral de las personas..." (Art. 71). Los profesorados brindados en el marco de los institutos superiores, se estructuran en tres campos de formación, siendo éstos el de la Formación General, Específica y de la Práctica. Los profesorados presentan una duración de 4 años, cuyo título obtenido al finalizar la carrera, habilita a los egresados a desempeñarse en un nivel y disciplina específica. En la Ley de Educación Superior N²4.521 en su artículo 6º se menciona que "la Educación Superior tendrá una estructura organizativa abierta y flexible, permeable a la creación de espacios y modalidades que faciliten la incorporación de nuevas tecnologías educativas", especificaciones normativas que debieron atenderse en las condiciones sanitarias acontecidas que exigieron la suspensión de la presencialidad en el dictado de clases y el necesario uso de los medios tecnológicos para la continuación de los procesos de enseñanza.

El Profesorado en Educación Secundaria en Matemática es una carrera de formación docente, en cuyo plan de estudio se encuentra "Sociología de la Educación", unidad curricular que integra el campo de la formación general la cual se ubica en el segundo año de la carrera, presentando una carga horaria de 7 horas semanales cuatrimestrales, siendo su régimen de cursado presencial. A través de esta asignatura, se busca que el futuro docente comprenda el marco sociocultural, político y económico que enmarca los desarrollos y problemas que atraviesa la sociología, pueda identificar los diferentes enfoques teóricos, como también, que el docente en formación, construya una mirada sociológica de la realidad educativa, como asimismo analice y comprenda las prácticas educativas que se llevan a cabo en este ámbito y las problemáticas que la atraviesan. 


\section{LA PROPUESTA DE ENSEÑANZA Y LAS ACTIVIDADES DE APRENDIZAJE}

La experiencia se centra en la propuesta de enseñanza de la asignatura Sociología de la Educación del Profesorado en Educación Secundaria en Matemática del Instituto de Educación Superior "Clara J. Armstrong". El uso del WhatsApp, permitió que el cursado sea accesible a la totalidad de los alumnos inscriptos, ya que varios no disponían de otro medio tecnológico que el celular y no contaban con wi-fi en sus hogares. Los estudiantes que participaron de la experiencia fueron 24, de los cuales 13 entregaron la totalidad de las actividades, 9 de forma parcial y 3 no participaron de la materia.

La formación del grupo de WhatsApp para la asignatura, significó como primera medida, la creación de un enlace por medio del cual se incorporaron los alumnos inscriptos en la materia, una vez formado, se realizó la presentación de la docente, la asignatura y la modalidad de trabajo. El grupo estaba constituido por docente y alumnos y su formación permitió que los estudiantes tuvieran acceso al conjunto de materiales que se utilizarían en la materia, el cual se enviaba en formato PDF. Las clases se compartían semana de por medio y consistían en una síntesis explicativa del tema y junto a éstas se adjuntaba las actividades asignadas a los estudiantes, las que en casos concretos se ejemplificaban para su mejor comprensión; la entrega de las actividades, también se realizaba semana de por medio, las que, dependiendo de la dificultad y el objetivo de la misma, se elaboraban de forma individual, en pareja o en grupos de tres. El medio de entrega de los trabajos por parte de los alumnos, fue en una oportunidad mediante una foto que debían subir al grupo, y las siguientes se llevaron a cabo a través del correo electrónico, adjuntando el trabajo en un formato accesible para el alumno (fotos, documento Word o PDF), lo que facilitó la participación y continuidad en la materia ya que no se exigió ningún formato especifico.

A través del grupo de WhatsApp se realizaba una devolución general de lo observado en los trabajos, como por ejemplo problemas en la redacción o faltas de ortografías, orientaciones sobre formalidades en la presentación de los trabajos, uso de términos propios de la disciplina en los escritos, etc., a estas observaciones se sumaban las correcciones particulares a cada grupo de los aspectos a atender o mejorar en cada actividad, lo cual se realizaban a través del correo electrónico.

Las clases proponían lectura de los materiales bibliográficos y la realización de actividades; en cada encuentro se ensayaban formas de trabajo que propiciaran diferentes procesos cognitivos, como relacionar conceptos y/o teorías con hechos de la realidad, recuperar saberes previos, argumentar, buscar información en distintas fuentes, elaborar, reflexionar, etc. Formó parte de las actividades de algunas clases, el observar videos recuperados de Youtube, los que en ocasiones se colgaban en el grupo y en otras se adjuntaba el enlace en el texto de las actividades. Las estrategias de enseñanza y las actividades se fueron definiendo clase a clase y su diseño estaba vinculado a las posibilidades que ofrecían los contenidos, y a la necesidad de generar en los estudiantes formas distintas de acercarse y elaborar los temas. Se buscó que "los estudiantes puedan trabajar en equipo, pensar de forma crítica y creativa y reflexionar acerca de su propio proceso de aprendizaje" 
(Organización de las Naciones Unidas para la Educación, la Ciencia y la Cultura [UNESCO], 2004).

A continuación, se describirán algunas de las actividades propuestas durante el desarrollo de la $1^{\circ}$ y $2^{\circ}$ unidad temática de la materia.

Una de las actividades de la unidad 1 tuvo como objetivo que los alumnos se introdujeran en el conocimiento de la sociología como disciplina científica, en esta ocasión se les planteó realizar una actividad individual, que consistió en realizar la búsqueda de definiciones e información general sobre sociología en distintas fuentes (diccionarios, libros de autor, internet, etc.), también, que observaran dos videos, y leyeran citas bibliográficas de autores diferentes. Tomando la información de las distintas fuentes, los alumnos debían redactar un texto explicativo breve, que versara sobre la Sociología. En la escritura del texto debían, de forma articulada, explicar que es la sociología, cuál es su objeto de estudio, qué indaga sobre la sociedad y el hombre, y que aportes realiza para la comprensión de la realidad social. La actividad descrita, demandó de los alumnos búsqueda y selección de la información, organización de las ideas y elaboración del texto, cuya redacción final requería por parte de los estudiantes la necesaria comprensión previa de la temática.

Otras de las actividades, también de la $1^{\circ}$ unidad, tuvieron como objetivo que los alumnos se acercaran a las definiciones de los conceptos adoptados desde la asignatura y que realizaran asociaciones con un hecho específico de la realidad social. La actividad consistió en solicitar a los alumnos que leyeran definiciones de sociedad citadas en la bibliografía, como también la definición que se adoptaba desde la cátedra, asimismo debían identificar los aspectos comunes entre las definiciones y luego reelaboraran las características (unión, intencional, estable, estructurada, hacia un fin común) propias de la definición de sociedad asumida por la materia, esto lo debían realizar con sus propias palabras. El trabajo de reescritura debía ir unido a la identificación de las características que se manifestaba con más fuerza en los tiempos de pandemia en Argentina, y la justificación de por qué creían que esto ocurría, para la realización de esta actividad también debía buscar un soporte visual (imagen) que representara lo expresado por ellos. El desarrollo de la actividad les planteó, la reelaboración del texto consultado, el establecimiento de conexiones entre los conceptos y hechos de la realidad, al igual que una justificación sobre la vinculación establecida, lo cual favoreció el desarrollo de una mirada sociológica en los alumnos.

Durante el desarrollo de la $2^{\circ}$ unidad, se les asignó a los alumnos la actividad de trabajar con la técnica de Mapa Cognitivo de Aspectos Comunes, en este caso el objetivo fue que los alumnos/as (resolución de a pares) establecieran relaciones entre temáticas de la unidad, en esta oportunidad debían realizar dos Diagramas de Venn con una intersección. En uno de los diagramas los alumnos tenían que consignar elementos teóricos del Paradigma Funcionalista, en el otro diagrama, hacían algo similar, pero con los postulados de la teoría de Emilio Durkheim y en la intersección debían consignar los puntos de encuentro que habían detectado entre ambos desarrollos. Esta actividad exigió que los alumnos realizaran una síntesis de los postulados más importantes de cada constructo 
teórico y que también en la intersección escribieran las relaciones conceptuales identificadas entre el paradigma y la teoría.

En vistas a ensayar formas de trabajo que superaran una problemática común entre los alumnos -la copia textual de la bibliografía para la resolución de las actividades- se describen dos actividades que obligaron a los estudiantes a escribir, pero siguiendo la regla de no copiar o escribir con palabras de uso cotidiano, pero sin alterar la propuesta teórica de los autores. Para lograr este objetivo se establecieron actividades diferentes, una de ellas fue escribir un comentario de las ideas postuladas por el paradigma de la Acción Social, en cuyo escrito solo debían consignar lo comprendido, sin hacer copia textual y respetando la extensión permitida (1 carilla), la segunda actividad fue elaborar una carta (siguiendo su estructura) a un familiar o amigo en donde explicaran de forma sintética, lo propuesto por Max Weber en su teoría. En los escritos se resaltaron aspectos diferentes, lo cual estuvo sujeto a lo comprendido por los estudiantes. Cabe aclarar que algunos de los trabajos fueron devueltos para su reelaboración, ya que muchos no cumplían con las normas de escritura estipuladas.

En otra clase, también de la $2^{\circ}$ unidad, la actividad consistía en realizar el esquema SQA, en la cual los alumnos debían recuperar lo que ya conocían sobre el tema Sistema Educativo, lo que les interesaba seguir aprendiendo del mismo (expresado mediante preguntas) y lo que habían descubierto mediante la lectura del texto. La tarea les demandó realizar identificación de sus saberes previos sobre el tema y también su redacción, elaborar interrogantes que describieran lo que querían seguir conociendo, y comentar lo develado de la lectura del material.

\section{RESULTADOS LA EXPERIENCIA DE LOS ALUMNOS Y EL USO DEL WHATSAPP EN LA ENSEÑANZA SEGÚN LA ENCUESTA}

Al finalizar el cuatrimestre, se les solicitó a los estudiantes que completaran una encuesta semi-estructurada, en donde se les consultó acerca de la experiencia de enseñanza y aprendizaje que se había llevado a cabo en la materia Sociología de la Educación. La encuesta fue compartida por el grupo de WhatsApp, y su devolución se realizó a través del correo electrónico. La encuesta contenía preguntas abiertas y cerradas con opciones múltiples. El instrumento se estructuró en base a dos categorías: 1) las plataformas utilizadas en la materia, utilidades que se les dio y otras que podrían implementarse y el grado de acceso a la propuesta de la materia. Con relación a la categoría 2) experiencia de enseñanza y aprendizaje de Sociología de la Educación, se buscó conocer la opinión sobre los modos de organización de las clases y las posibilidades de seguir un hilo temático a partir de su estructura, las actividades y su realización en función del tiempo y su complejidad, el trabajo cognitivo que les demandó su resolución, la evaluación y la forma de llevarse a cabo, también se les solicitó una valoración numérica sobre la experiencia transitada en la materia y aportes que permitieran mejorar la propuesta. Las respuestas coincidieron en que el uso del WhatsApp, fue la plataforma de trabajo que les permitió a todos los estudiantes el cursado de la materia y acceder por este medio a los contenidos curriculares y a su

\section{Maciel Ortega}


propuesta de enseñanza y aprendizaje, y que el modo de estructurar el texto de las clases, fue orientador para seguir los temas de la materia.

En cuanto a las actividades, los estudiantes refirieron que estaban planteadas de forma clara y que eran realizables en los tiempos asignados, y que en su generalidad contribuyeron a un acercamiento comprensivo de los temas, trabajar en grupo, intercambiar opiniones, investigar en distintas fuentes y relacionar contenidos con hechos de la realidad, lo cual pone en evidencia que desde la propuesta de cátedra se buscó "el desarrollo de nuevas y desafiantes propuestas de formación que pongan en diálogo y tensen los saberes aprendidos con las nuevas problemáticas (...) contemporáneas" (Arata, 2020, p. 67), entre otros.

Cuando se les solicitó a los estudiantes información sobre que otras estrategias podrían haberse implementado desde el WhatsApp, los alumnos mencionaron que les hubiera gustado acceder a videos donde se pudiera ver y escuchar a la docente explicando la materia. Es dable señalar, que la presentación explicativa de los contenidos formaban parte del material que se elaboraba clase a clase y también de los audios compartidos, aunque lo solicitado por los estudiante conduce a suponer que debido a la no presencialidad física de la docente frente al aula, una proyección donde se la pudiera ver o una clase mediada por plataformas que permitieran la sincronicidad y la interacción, podría haber reemplazaba tal ausencia, situación que pese a tratarse de un nivel superior, pone en evidencia el valor de la presencia física del docente en el proceso de enseñanza y del encuentro comunicativo cara a cara.

Los resultados de la intervención fueron valorados como positivos, aunque solo 7 estudiantes respondieron a la encuesta.

La experiencia de enseñanza mediada en este caso por una aplicación de WhastApp requirió establecer nuevas formas de trabajo y de comunicación pedagógicas, y establecer un viraje en cuanto al trabajo del docente, ya que la tarea implicó desistir de "ser únicamente el de transmisor de conocimiento para convertirse en un facilitador y orientador del conocimiento y en un participante del proceso de aprendizaje junto con el estudiante" (UNESCO, 2004).

\subsection{RESULTADOS}

El WhatsApp en el caso particular de esta experiencia, fue usado como medio a través del cual se construyó el vínculo con los alumnos y se estableció una comunicación tanto sincrónica como asincrónica, modos de interacción que operaron cambios tanto a nivel de la relación docente-alumnos como de la enseñanza.

A la aplicación se le asignaron "usos estrictamente pedagógicos [los cuales] tienen vinculación directa con los contenidos curriculares correspondientes a las asignaturas del curso" (Casablancas, 2018, p. 160); se la utilizó como el canal para el desarrollo explicativo de los temas y la administración de actividades y la dotación de recursos bibliográficos y audiovisuales, el espacio a través del cual se compartía información, se establecían 
acuerdos entre profesor y alumnos y se realizaban observaciones generales sobre los aspectos a mejorar en los trabajos que se elaboraban. EL "contrato pedagógico (...) enmarcó las modalidades de uso tecnológico" (Op. cit. 2018, 172) que se haría del grupo de WhatsApp en el marco de la asignatura.

Como ya se hiciera mención en otras partes del trabajo, esta plataforma permitió el acceso a la propuesta de enseñanza y aprendizaje diseñada desde la cátedra a la totalidad de los alumnos inscriptos en la materia, posibilitó la inclusión del estudiantado en la propuesta diseñada ya que el conjunto de alumnos disponía de esta aplicación en sus teléfonos celulares, no así de computadoras ni servicio de internet, que de haber sido así, se hubieran podido emplear de manera complementaria o alternativa otras plataformas virtuales que ofrecieran formas de cursado diferentes, pudo observarse de modo directo en este grupo de alumnos, la existencia de una brecha digital, la cual se caracterizó por la desigual tenencia y conocimiento sobre el uso y los beneficios que brindan los medios tecnológicos a nivel de la enseñanza y el aprendizaje. "La brecha digital provoca que personas, sectores sociales y naciones vivan en condiciones desfavorables porque se ven impedidas al acceso y uso de la información, comunicación y conocimiento" (Butrón Untiveros, 2018, p. 189).

La generalidad de las actividades propuestas en cada clase, buscaron que el/los alumnos llevaran a cabo procesos cognitivos diferentes y que desarrollaran prácticas de lectura y escritura variadas, como también la construcción de aprendizajes centrados en las temáticas trabajadas en cada clase, se aspiró a que "las acciones externas se transformen en acciones mentales internas" (Riestra, 2008, p. 128). Se tendió a "ensayar propuestas formativas novedosas teniendo presente y reconociendo la heterogeneidad de poblaciones, (...), al mismo tiempo que se [buscó] garantiza[r] que las propuestas formativas lleg[aran] a todos y todas por igual. (Op. cit. 2020, p. 68)

Los alumnos que participaron activamente en la propuesta de la materia, mostraron poder resolver las actividades sin dificultad, aunque con mucha dependencia y copia textual de la bibliografía, siendo las actividades de escritura autónoma el talón de Aquiles del grupo, ya que en varias oportunidades las tareas fueron devueltas para su reelaboración. Las producciones que los alumnos presentaban en cada clase y las respuestas vertidas por ellos en la encuesta de valoración de la experiencia educativa en el marco de la asignatura, mostraron que, pese a la fuerte dependencia respecto de los textos, los alumnos comprendían los contenidos, interpretaban las consignas, podían establecer relaciones entre conceptos y elaboraban los trabajos siguiendo las pautas establecidas en los encuentros y que las actividades contribuían al ejercicio de procesos de pensamiento específicos y mayores niveles de autonomía en su resolución. Un aspecto observado en la generalidad de los estudiantes fue, que los alumnos podían construir "el sentido [de los] texto[s] (...) a partir de sus propios conocimientos y de su intensión de lectura..." (Cubo de Severino, 2005, p. 212), pero mostraban dificultades al comunicar de modo escrito lo interpretado, mediante la utilización de términos de uso cotidiano. 
Cabe aclarar que las actividades dispensadas al grupo de alumnos fueron devueltas con una valoración cualitativa (aprobado; desaprobado, rehacer o corregir), ya que por Resolución N³63 del Consejo Federal de Educación, la evaluación debía adoptar un carácter formativo y apuntar a retroalimentar los procesos de enseñanza y aprendizaje y acompañar en la trayectoria del estudiante mientras durara la pandemia.

El proceso de enseñanza y aprendizaje no presencial, demandó a los alumnos la construcción de una disciplina de lectura y estudio constante, que al momento de llevarse a cabo en la materia (en tiempos de pandemia), no todos los estudiantes la tenían desarrollada, lo que les acarreó un esfuerzo mayor el sostenimiento en el cursado de la asignatura y la carrera elegida.

Es pertinente mencionar que quedaron actividades sin desarrollar por finalización de cuatrimestre, las cuales eran posible de realizar mediante la aplicación y uso del celular como fueron los foros de opinión y la grabación de videos o audios explicativos elaborados por alumnos, que pudieran compartirse por el grupo.

\section{DISCUSIÓN DE LOS RESULTADOS}

Investigaciones realizadas en otros contextos institucionales, centradas en el uso pedagógico-didáctico del WhatsApp, mostraron resultados similares a los logrados en Sociología de la Educación, uno de estos trabajos fue el realizado por Omar J. Trejo Buritica, en la asignatura Programación II de la carrera de Ingeniería en Sistema, titulada "WhatsApp como herramienta de apoyo al proceso de enseñanza y aprendizaje de la programación de computador". Los resultados mostraron que el uso pedagógico de la aplicación mejora los procesos de aprendizajes en programación, ya que posibilita el desarrollo de la comunicación y el planteo de consultas de forma directa al profesor, y la obtención de respuestas en diferentes formatos como los videos, las fotos, los audios y los mensajes escritos.

Por otra parte el trabajo "WhatsApp: su uso educativo, ventajas y desventajas", llevado a cabo por Belén Suárez Lantarón, mostró que en la enseñanza de nivel superior es donde más se utiliza la aplicación como herramienta pedagógica, la cual presenta como beneficios, una fluida comunicación e intercambio de información y materiales entre docentes y alumnos, asimismo porque oficia como espacio de motivación para el aprendizaje de los estudiantes, puede ser usado en la educación formal como informal y en la de carácter on line, y en diferentes disciplinas, ya que permite la participación de todos a partir de dudas, reflexiones, comentarios, etc. Entre las desventajas atribuidas a la aplicación se encuentran que los mensajes pueden ser enviados en horarios inadecuados y que estos en algunos casos pueden ser interpretados de forma inadecuada y que los participantes pueden compartir contenido no académico. En lo referente a este último aspecto, no fue algo que ocurriera en el grupo de la materia Sociología de la Educación, ya que se estableció como regla que las consultas se debían realizar de lunes a viernes y en determinados horarios, y que el grupo sería de carácter académico, aunque si se recupera como un aspecto a mejorar 
que las consultas de los estudiase se realizaran por el grupo y no como ocurría frecuentemente por mensajes privados.

Por último, el trabajo realizado por Carmen J. Pradón, titulado “Estrategias didácticas basadas en aplicaciones de mensajería instantánea WhatsApp exclusivamente para móviles (mobile learning) y el uso de la herramienta para promoción del aprendizaje colaborativo" , los resultados obtenidos evidenciaron que la integración del WhatsApp a las estrategias formativas permite la construcción de conocimientos de forma colaborativa entre los grupos y la mejora de la comunicación ya que posibilita el desarrollo de tutorías, el recordatorio de entrega de trabajos, el intercambio de imágenes, música, videos, audios, entre otros, situación que coincide con los usos dados a la aplicación en el marco de la experiencia sistematizada.

\section{CONCLUSIONES}

La enseñanza de Sociología de la Educación a través del WhatsApp y el correo electrónico, puso en evidencia que, aunque la aplicación y el mail no son plataformas que hayan sido creada con fines pedagógicos, sus utilidades pueden ser adaptadas para que cumplan este fin, y que la incorporación de medios tecnológicos en la enseñanza, por sí mismos no generan innovación ni aprendizaje sino que las estrategias de enseñanza propuestas y las actividades diseñadas, son las que favorecen o no la construcción de saberes.

Se pudo constatar que el uso de diferentes plataformas, también requiere formas de trabajo diferentes a las que demanda la presencialidad, ya que solicita el diseño de actividades que potencien la búsqueda de información (escrita, audiovisual) que permitan el esclarecimiento de dudas, el acceso a nuevos enfoques conceptuales, que abran la posibilidad de encontrarse con fuentes e información variada, y hasta distinta a las ofrecidas desde la cátedra, potenciando así la construcción de posicionamientos propios, similares o alternativos a los presentados.

Como la descripción de la experiencia lo demuestra, las actividades eran diferentes en cada clase, en su mayoría exigían la lectura de materiales bibliográficos y variados ejercicios de escritura, que demandó el ensayo de expresar las ideas de forma organizada y clara, reafirmando lo comprendido de los documentos, hecho que puso en evidencia problemas de redacción y una fuerte dependencia respecto de los textos y las palabras de los autores.

El trabajo de enseñanza y aprendizaje no presencial, impuso a los estudiantes la formación de un hábito de lectura y estudio constante, que al momento de desarrollarse en el nivel superior (en tiempos de pandemia), no todos los estudiantes lo tenían incorporado, lo que les demandó a algunos un esfuerzo extra el sostenimiento en el cursado de la asignatura y a otros optar por abandonar el cursado (de forma momentánea o permanente?) y realizarlo cuando se retornara a la presencialidad.

La enseñanza a través de medios virtuales, puso en evidencia la necesidad de establecer lazos comunicacionales tanto sincrónicos como asincrónicos con los estudiantes, que reforzaran los vínculos y posibilitaran la consulta y evacuación de dudas, y que la mediación 
tecnológica requiere de un trabajo de redefinición y selección de los contenidos a desarrollar ya que no todos los temas permiten ser desarrollado a través de estos canales.

Cuando se les planteó a los estudiantes que otras plataformas podrían haberse usado, si todos hubieran tenido computadoras y servicio de internet, los alumnos respondieron que podría haberse implementado el aula virtual de Classroom, plataformas como Edmodo y Zoom, los cuales de haberse dado la tenencia de los medios necesarios por parte de los alumnos, hubiera permitidito encuentros sincrónicos semanales y un reconocimiento por parte de los alumnos respecto de la figura del docente, y viceversa.

La sistematización y análisis de la experiencia permitió construir un conocimiento sobre las posibilidades y límites que impone el WhatsApp como medio tecnológico aplicado a la enseñanza, como también reconocer la necesidad de implementar en nuevas experiencias otros medios y formas de trabajo que incluyan como parte de los contenidos a enseñar y aprender el uso de nuevas tecnologías, ya que esto fortalecería la formación de los futuros docentes y permitiría al mismos tiempo, poder llevar a cabo la construcción de otro tipo de estrategias metodológicas.

\section{REFERENCIAS}

Arata, N. (2020). La escuela frente a la pandemia. Entre la defensa de lo común y la búsqueda de alternativas. En I. Dussel; P. Ferrante y D. Pulfer (compiladores), (2020). Pensar la educación en tiempos de pandemia. Entre la emergencia, el compromiso y la espera. UNIPE: Editorial Universitaria.

Butrón Untiveros, E. (2018). Una computadora por alumno, experiencia por decreto. En S. Lago Martínez; A. Álvarez; M. Gendler y A. Méndez (Editores), (2018). Acerca de la apropiación de tecnologías. Teoría, estudios y debates. Ediciones del Gato Gris.

Casablancas, S. (2018). Nuevas tendencias de comunicación y participación en las escuelas 2.0. En S. Lago Martínez; A. Álvarez; M. Gendler y A. Méndez (Editores), (2018). Acerca de la apropiación de tecnologías. Teoría, estudios y debates. Ediciones del Gato Gris.

Cobo, C. (2016). La Innovación Pendiente. Reflexiones (y Provocaciones) sobre educación, tecnología y conocimiento. Colección Fundación Ceibo.

Coll, C.; Mauri Majós, M. y Onrubia Goñi, J. (2008). Análisis de los usos reales de las tic en contextos educativos formales: una aproximación sociocultural. Revista Electrónica de Investigación Educativa, 10 (1). http://redie.uabc.mx/vol10no1/contenido-coll2.html

Cubo de Severino, L. (2005). Leo pero no comprendo. Estrategias de comprensión lectora. Comunicarte Lengua y discurso.

España, A. y Foresi, M. (2009). Las prácticas y el desarrollo profesional ante las nuevas tecnologías de la información y de la comunicación. En L. Sanjunjo (Coord.), Los 
dispositivos para la formación en las prácticas profesionales (pp. 183-222). Homo Sapiens.

Edelstein, G. (1998). Un capítulo pendiente: el método en el debate didáctico contemporáneo. En G. Edelstein Corrientes didácticas contemporáneas (p. 75-89). Paidós.

Libedinsky, M. (2106). La documentación de experiencias educativas innovadoras. En M. Libedinsky La innovación educativa en la era digital (pp.85-98). Paidós.

Maggio, M. (2016). Enriquecer la Enseñanza. Paidós.

Maggio, M. (2018). Reinventar la clase de la universidad. Paidós.

Organización de las Naciones Unidas para la Educación, la Ciencia y la Cultura (2004). Las tecnologías de la información y la comunicación en la formación docente. Guía de planificación. TRILCE. https://pide.files.wordpress.com/2007/08/documento-unescotic-y-formacion.pdf

Padrón, C. J. (2013). Estrategias Didácticas basadas en Aplicaciones de Mensajería Instantánea WHATSAPP exclusivamente para Móviles (Mobile Learning) y el uso de la Herramienta para promover el Aprendizaje Colaborativo. Dialnet, 7(2),123-134.

https://dialnet.unirioja.es/servlet/articulo?codigo=4857401

Riestra, D. (2008). Las consignas de enseñanza de la lengua. Un análisis desde el interaccionismo-discursivo. Miño y Dávila.

Suárez Lantarón, B. (2018). Whatsapp: su uso educativo, ventajas y desventajas. Revista de Investigación en Educación, 16(2), 121-135 http://webs.uvigo.es/reined/

Resolución No 108 de 2020. [Ministerio de Educación Nacional]. Suspender el dictado de clases presenciales en los niveles inicial, primario, secundario en todas sus modalidades, e institutos de educación superior, por (14) días corridos a partir del 16 de marzo. 15 de Marzo de 2020.

Resolución No 363 de 2020 [Consejo Federal de Educación]. Aprobar, las "Orientaciones para los procesos de evaluación en el marco de la continuidad pedagógica" que constan en el Anexo I y aprobar la realización de la "Evaluación nacional del proceso de continuidad pedagógica", que consta en el Anexo II. 15 de Mayo de 2020.

Trejos Buriticá, O. I. (2018). WhatsApp como herramienta de apoyo al proceso de enseñanza y aprendizaje de la programación de computadores. Dialnet (35), 149-158. https://dialnet.unirioja.es/servlet/articulo?codigo $=6702430$ 